\title{
'La audiencia no lo es todo'. Cuota de pantalla frente a calidad percibida: una propuesta metodológica
}

\author{
Francisco Alberto Vallejo PeÑA \\ Universidad de Málaga \\ favallejo@uma.es \\ Andrés Villena Oliver \\ Universidad de Málaga \\ villenaoliver@uma.es
}

Recibido: 8 de octubre de 2014

Aceptado: 20 de enero de 2015

\begin{abstract}
Resumen
La presente investigación aborda un análisis del actual escenario televisivo español con el objeto de identificar y caracterizar la distribución de las audiencias por canales, así como la valoración que estos obtienen de sus espectadores. El marco teórico resalta, ya desde autores clásicos como Merton y Bourdieu, que la búsqueda de las audiencias de masas se lleva a cabo compitiendo con productos de escasa calidad. De hecho, los datos presentados muestran cómo algunas de las cadenas más vistas obtienen una baja valoración por parte de sus espectadores. En base a ello se desarrolla, como propuesta metodológica, un índice que pondera la cuota de pantalla de las cadenas en función de la valoración realizada por sus propios televidentes (Índice de Audiencia Relativa). Tras su aplicación a los grupos mediáticos Atresmedia y Mediaset, se concluye que el primero de ellos está logrando mejores resultados en audiencia, cuando esta se pondera por la imagen generada.
\end{abstract}

Palabras clave: competencia mediática, telebasura, cadenas de televisión, programación de calidad, tv channels, mercado audiovisual.

\section{'Questioning audience data'. TV share vs. viewers' aluation: a methodological proposal}

\begin{abstract}
This research consists of an analysis of the Spanish television context to characterize the current distribution of the main channels' audiences, as well as the assessment or valuation from viewers. Theory has emphasized, from classic authors like Merton or Bourdieu, that competition for mass audiences takes place leaving aside the quality criteria. In fact, some of the most shared channels obtain a low valuation from its viewers. On this basis, we propose a method that provides an index to measure the audience according to the valuation achieved (Relative Audience Index). Then, the index is used to compare Atresmedia's results with Mediaset's. In conclusion, Atresmedia is achieving best audience results when viewers' valuation criteria are taken into account.
\end{abstract}

Keywords: media competition, trash TV, quality programming, TV channels, audiovisual market.

\section{Referencia normalizada}

VALLEJO PEÑA, Francisco Alberto y VILLENA OLIVER, Andrés (2015): “'La audiencia no lo es todo'. Cuota de pantalla frente a calidad percibida: una propuesta metodológica". Estudios sobre el Mensaje Periodístico. Vol. 21, Núm. 2 (julio-diciembre), págs.: 1275-1293. Madrid, Servicio de Publicaciones de la Universidad Complutense.

Sumario: 1. Planteamiento de la investigación: la disociación entre audiencia y calidad. 2. Antecedentes teóricos; 2.1. La televisión como actor empresarial; 2.2. Acerca de los efectos negativos de la televisión y del papel de las instituciones. 3. Materiales y metodología. 4. Validación del índice. 5. Resultados y análisis; 5.1. Descripción previa del panorama televisivo en audiencia e índice de imagen; 5.2. Aplicaciones del Î́ndice de Audiencia Relativa. 6. Conclusiones. 7. Agradecimientos. 8. Referencias bibliográficas. 


\section{Planteamiento de la investigación: la disociación entre audiencia y calidad}

Una de las principales características del presente período es, sin duda, la gran capacidad de influencia de los medios de comunicación de masas, entre los que destaca la televisión. La envolvente presencia de este medio condiciona notablemente nuestro proceso de socialización, así como nuestras relaciones sociales y formas de ocio. Estas realidades contrastadas nos llevan a interesarnos por el impacto de sus disfunciones en las últimas décadas, reiteradamente señaladas por las innumerables denuncias de la sociedad civil en todo el mundo. A pesar de las enormes posibilidades que el fenómeno televisivo supone desde su origen, en la actualidad, los programas de mayor calidad conviven con productos socialmente nocivos (Lacalle, 2000; Elías, 2004). Además, el peso de estos últimos ha arrastrado al conjunto televisivo a un estereotipo difícil de contrarrestar.

El fenómeno de la telebasura tuvo sus inicios con los realities estadounidenses de los 70, dando lugar a que la prensa acuñara él término (trash tv) a principios de los 80 (Murray y Oullette, 2009). Tras un rápido contagio en el mundo occidental se desarrolla un modelo televisivo mixto (privado-público) que conduce a que la calidad televisiva y la audiencia tiendan a situarse y medirse en distintas dimensiones. Este escenario se configura, en el caso español, en la década de los 90. Los crecientes intereses empresariales intensifican la competición por alcanzar el mayor porcentaje de audiencia posible (Vallejo Peña, 2012). La presión de los resultados a corto plazo conduce a una progresiva disociación y separación entre el objetivo de difusión masiva y la calidad televisiva. La parrilla se vuelve cada vez más sensible a las respuestas del público, por lo que tienen lugar continuos cambios de programa, formatos y horarios de emisión. La televisión pública, a su vez, se ve arrastrada por el nuevo modelo y adapta sus patrones. El sector público está desde entonces ante un difícil reto: soportar la presión hacia la calidad exigida por las instituciones y los contribuyentes y, además, generar resultados competitivos que le garanticen cierta presencia.

En este contexto el producto de calidad encuentra grandes dificultades para permanecer en las parrillas, con el desestímulo que esto implica para sus creadores. En respuesta surgen nuevas fórmulas, como la televisión segmentada y especializada desde los primeros 2000, que se concibe para grupos pequeños y, en algunos casos, de pago. Esta tendencia abre nuevos horizontes y permite eludir algunas disfunciones propias del producto de masas, aunque presenta algunas limitaciones: (1) su carácter excluyente de audiencias de bajo perfil cultural y/o económico, (2) además de las consecuentes dificultades para financiarse (Villena, 2005).

La tendencia a tal disociación entre audiencia y calidad se observa actualmente en la imagen percibida por el público de los canales televisivos en España (Índice GECA -Grupo de Estudios de Comunicación Audiovisual-, 2012), que presenta valores distantes a la cuota de pantalla alcanzada en sus emisiones. En este sentido, un estudio de la distribución de las variables índice GECA y cuota de pantalla muestra que estas no establecen precisamente una relación lineal. Por tanto, al comparar la cuota de pantalla de los canales televisivos, obtendremos algunos indicadores sobre su poder e influencia social relativa; sin embargo, estos datos no informarán sobre la valoración que de la calidad del producto realiza el público. De hecho, a pesar del liderazgo ejercido 
por la cadena Telecinco en cuota de pantalla, el canal de Mediaset presenta la valoración más pobre de la audiencia entre las cadenas generalistas (datos comentados ampliamente en los resultados de este trabajo). En el mercado actual lo realmente difícil para un productor televisivo no es lograr grandes audiencias, sino hacerlo a la vez que se obtienen niveles de calidad razonables -lo cual repercute en la capacidad de fidelización del público y, por lo tanto, en la generación de un alto nivel de audiencia futura-. Además, el equilibrio entre audiencia y calidad tiene una gran importancia para cumplir criterios de responsabilidad social:

"Los promotores de la RSC deberán desarrollar los mecanismos de protección que eviten que los medios nocivos encuentren en ella la capa de maquillaje que facilite su divulgación institucional y comercialización. Asimismo, deberán desarrollarse instrumentos de medida en RSC especializados en el campo de medios de comunicación, que cuenten con especial sensibilidad para reflejar las particulares repercusiones sociales que implica el sector" (Vallejo Peña, 2012: 121).

En base a estos planteamientos, el objetivo principal de este trabajo es la presentación de una propuesta metodológica para estudiar la audiencia de los distintos canales de televisión, ponderando sus valores en función de los niveles de calidad alcanzados. Posteriormente, se aplicarán tales mediciones a los dos principales grupos televisivos privados en España (Mediaset y Atresmedia), con objeto de valorar y comparar su reciente desempeño en estos aspectos.

\section{Antecedentes teóricos}

\subsection{La televisión como actor empresarial}

Detrás de la mayoría de las emisiones televisivas existe un entramado de decisiones empresariales que han de tenerse en cuenta para una mejor comprensión del objeto de estudio. Estas circunstancias, junto con los avances impulsados por la denominada revolución tecnológica y la creación de grandes consorcios mediáticos, han llevado a algunos analistas a recomendar que se conciba la información prioritariamente como una mercancía (Ramonet, 1998). Desde este punto de vista, resulta razonable observar y analizar una empresa televisiva como otra cualquiera en sus aspectos básicos. Para Varian, las empresas "son actores racionales que tratan de maximizar sus beneficios elevando en lo posible sus ingresos y rebajando sus costes. Ambas vías no son excluyentes; es más, combinadas producen el máximo beneficio perseguido" (Varian, 2005: 329). Bustamante, por su parte, resalta el peso que el negocio de la publicidad tiene sobre la gestión de las grandes televisiones privadas: "El objetivo indudable de la televisión privada, abierta y gratuita es [...] conseguir la máxima inversión publicitaria $\mathrm{o}$, dicho en términos de cualquier empresa en una sociedad capitalista, maximizar sus beneficios" (Bustamante, 1999: 72). Para ello, la televisión transforma la audiencia producida por sus programas en espacios para la inserción de publicidad, que esta vende a unos determinados anunciantes de tal modo que "el operador (la televisión) se constituye en auténtica máquina de transformar inversiones en programas en ingresos sacados de la audiencia de estos programas" (1999: 21).

Las consecuencias de este modelo productivo y financiero se materializan con mayor claridad en los realities y en los talk shows. Acerca de este último formato te- 
levisivo, Villena (2005) resalta el propósito de obtener ingresos a partir de costes bajos recurriendo a un conjunto de invitados que fueron escogidos por ser previamente etiquetados como "culturalmente vulnerables". Estos individuos se caracterizan por tener pocos ingresos, dificultades formativas y laborales, así como un bajo nivel educativo (frecuentemente reflejado a través de problemas de expresión lingüística). Estas cuestiones no constituyen obstáculo alguno para que se genere un espectáculo capaz de lograr altas audiencias (Lacalle, 2000). Todo ello, gracias a la construcción de historias impactantes, emocionales, conflictivas y, en muchos los casos, íntimas. Consideraciones éticas aparte, los medios de masas jamás han encontrado productos tan económicos que les permitan acceder a un gran número de espectadores.

Bueno (2003) destaca que, en la actualidad, se están dando dos formas diferentes de telebasura. La primera de ellas es la "fabricada": aquella que, sin pretenderlo, acaba generándose como tal en su afán de seducir a grandes audiencias. Su encarnación más clara es "Operación Triunfo" que, pese a sus pretensiones iniciales, representa la recreación de un "atajo profesional" de tres meses hacia la fama. En segundo lugar, define la "telebasura desvelada", es decir, la que se muestra dispuesta a poner en escena los ingredientes necesarios para conectar con una audiencia masiva ("Gran Hermano" es su más claro exponente). Bueno subraya el efecto perjudicial que estas prácticas están causando a nuestra socialización y a nuestro sistema de valores. En esta misma línea se pronuncia Elías (2004), que describe lo que denomina un "efecto contagio": el mencionado modelo televisivo cuenta cada vez más con la complicidad del periodismo serio, además de con otras instituciones como la propia Universidad, que colabora en el marco de unas prácticas de empresa que no dignifican precisamente la profesión periodística. Estos fenómenos conllevan a que la frontera entre la telebasura y la televisión de calidad se esté difuminando progresivamente.

Las características del modelo televisivo descrito nos conducen a buscar explicaciones como las ofrecidas por Coleman (1990) en su "teoría de la elección racional". El autor explica la dinámica y la evolución de los sistemas sociales a partir de la existencia de un conjunto de actores que compiten por el control de un recurso escaso y enormemente valioso (tiempo del espectador), que le permite obtener ingresos de los anunciantes, su fuente principal de financiación y, por lo tanto, sobrevivir. De esta forma, los actores mediáticos afrontan las estrategias de sus competidores en función del mantenimiento de su nivel de audiencia y de sus beneficios, lo que influye determinantemente sobre los contenidos finalmente ofrecidos.

Al reflexionar sobre estos fenómenos, la ciencia social tiende a presentar al ciudadano como un actor manipulable ante los medios que, además, no es defendido por su gobierno ni por el resto de las instituciones. Para interpretar el fenómeno, Moragas recurre a los argumentos de la "teoría de los usos y gratificaciones". Lazarsfeld formuló dicha teoría en los años 40 para interpretar las necesidades que cubrían los exitosos seriales radiofónicos de la época (Moragas, 1982). En los 70 -ya con la expansión de la televisión-, Katz y sus colaboradores Gurevich y Haas argumentaron que el uso que el ciudadano hace de los medios depende de la interacción de factores como su disposición psicológica, su ubicación social, su integración y determinadas pautas de selección (Katz et al., 1973). Además, dichos autores identificaron los cinco tipos de 
necesidades que cubrían los medios: cognitivas, afectivas, integradoras personales, integradoras sociales y evasivas. Por ende, el producto televisivo sólo debe encontrar su ubicación entre ellas, y será suficiente, por ejemplo, que sirva al ciudadano de válvula de escape de su propia realidad para permanecer en la parrilla.

\subsection{Acerca de los efectos negativos de la televisión y del papel de las instituciones}

La amenaza que implicaba para la humanidad el desarrollo de los medios de comunicación de masas ya fue denunciada por Lazarsfield y Merton en su trabajo publicado en 1977, Comunicación de masas, gusto popular y acción social organizada. Los autores reflexionaban sobre el perjuicio que los nuevos medios estaban generando en la sociedad norteamericana de los 70, dado que el interés económico se imponía claramente a sus fines sociales. Cuatro décadas más tarde, existe un amplio consenso sobre los perjuicios que la evolución de los medios y, especialmente, el fenómeno de la denominada "telebasura" han generado.

El imperativo del beneficio influye en lo que Berger y Luckmann denominan la "construcción social" de una realidad con la que entran en contacto de manera continua los ciudadanos y consumidores (Berger et al., 1967). La trascendencia económica de la actividad televisiva conduce a un cierto modo de producción icónico, al que remite Baudrillard con su idea de "la precesión de los simulacros" (Baudrillard, 1978). Para dicho autor, en una sociedad saturada de información e imágenes, son estas construcciones simbólicas las que se constituyen como la realización definitiva de los hechos sociales, de los que el consumidor es participante y testigo cotidiano. Ibáñez ya planteaba en los años 80 la importancia de la influencia de los grandes medios de masas en nuestra socialización, destacando que la verdad mediática se convierte, de alguna manera, en la verdad de todos (Ibáñez, 1985). En este mismo sentido, Mc Pherson, con su "teoría de las normas culturales", resalta la capacidad de los medios para transmitir ideas y valores de forma interesada y tergiversada al conjunto de la sociedad (García Ferrando, 1998). En esta línea se ha pronunciado también Bordas (2005) en su trabajo La sociedad caleidoscópica actual. Para el autor español, los medios de comunicación han logrado tal deformación de la realidad que -por destacar un ejemplo- los habitantes de las grandes ciudades del mundo han llegado a percibir una mayor probabilidad de ser víctimas de un delito de la realmente existente. En definitiva, se plantea un escenario de confrontación de la verdad mediática frente a la real, en la que la primera se impone a la segunda (2005: 197).

Bourdieu (1998) añade a través de su clásico On tv su perspectiva sobre la naturaleza espectacular y económica de la televisión. Desde su punto de vista, la búsqueda de la audiencia masiva conduce a la producción bajo el principio del máximo común denominador. Se libera, entonces, una competencia "por lo bajo", en la que el término calidad pierde relevancia. Las parrillas televisivas se han llenado de programas que cualquiera puede entender; paradójicamente, esta democratización del acceso ha reducido el margen para utilizar la televisión como vía de difusión de información de calidad y con cierta utilidad ciudadana. Bueno (2003) denuncia que este fenómeno, secundado por otros hechos que se han producido paralelamente, ha derivado en un público cada vez más ágrafo e inabordable desde la perspectiva del conocimiento. 
Por los diversos condicionantes anteriormente expuestos, los mensajes televisivos tienden a dirigirse a los valores y a las emociones del público potencial. Un reciente estudio sobre el papel de las emociones en el proceso de recepción y en la conciencia de la audiencia mostraba la vulnerabilidad de los españoles ante los medios: "Es 1lamativa la incapacidad de decodificación emocional por parte de la audiencia española [...] La interpretación que realiza la mayor parte de los sujetos es muy simple y sesgada, por lo que hemos podido comprobar que la audiencia es poco crítica y está escasamente alfabetizada en este indicador" (Lazo et al., 2013: 127). En líneas posteriores, los investigadores resaltan el papel que la educación mediática debe presentar en el futuro. Se trata de una herramienta esencial para que la población sepa "filtrar los contenidos como mecanismo de defensa ante la posible vulneración de sus derechos como usuario" (2013: 127).

Dado el grado de vulnerabilidad receptiva que han dejado estas lagunas en alfabetización mediática, el "entrenamiento de la audiencia" y la captación de su fidelidad se logra realmente a través del condicionamiento de nuestras emociones (conflictos, catástrofes o sucesos criminales, por ejemplo) (Fog, 2004). Estas son sencillas estrategias que resultan eficaces para llamar nuestra atención. De esta manera, con el apogeo de las televisiones privadas, se ha generado un escenario en el que se compite a través de la espectacularidad, generando contenidos de perfil bajo, aunque de gran eficacia para aumentar la audiencia. Un reciente estudio sobre la audiencia española de la serie "Friends" (Grandío, 2009) asociaba su éxito a factores como la gratificación inmediata (que resulte entretenido), la presentación de un submundo atractivo y la identificación con los personajes. Todo ello, por encima del ingenio de sus guiones. La autora subraya el dominio de la audiencia que los medios ejercen a través del "liking" (gusto basado en la seducción de nuestras emociones), pon encima del "taste", de connotación intelectual.

Estas circunstancias generan un escenario de indefensión consentida del ciudadano. En el ya señalado estudio de Lazo (2013), los mismos televidentes que indicaron que un anuncio no podía influir sobre sus decisiones individuales de compra (solo un $6,4 \%$ admitió que "sí"), contestaron más tarde que sí influirían notablemente en la decisión de compra de los demás $(82,6 \%)$. Estos resultados sugieren que afrontamos un escenario en el que la ingenuidad del público facilita su explotación económica por parte de las empresas.

Nuestro sistema democrático puede resultar muy eficaz para resolver grandes conflictos de Estado, o incluso internacionales. Sin embargo, la legislación vigente imposibilita combatir los contenidos menos funcionales de la televisión, así como sus efectos sobre la socialización de los ciudadanos. Nos encontramos en una etapa en la que la sociedad asume, de manera más o menos consciente, las "externalidades negativas" (Coleman, 1990) del fenómeno televisivo. Para Doyle (2002), no se trata de una cuestión de incompetencia política: las enormes dimensiones alcanzadas por los medios y su capacidad para controlar determinados sectores y recursos han conducido a una situación en la que los intereses de los televidentes sólo son defendidos en sus puntos de coincidencia con los productores y sus patrocinadores. Los intereses del consumidor que se quedan fuera de esta zona de coincidencia son, a la larga, nin- 
guneados y despreciados (McManus, 1995: 331-335). Un estudio reciente muestra que la actividad de las televisiones privadas en investigación e intervención sobre audiencias infantiles en España es inexistente, mientras que las televisiones públicas vienen desarrollando puntuales actuaciones que pretenden cuidar la socialización televisiva del público infantil. Las conclusiones del trabajo sólo salvan a TVE por su reciente actividad en este campo (Ortiz, 2013: 137).

En el ámbito del control televisivo institucional, España se encuentra actualmente en una situación anómala en el entorno europeo, siendo la única nación de la antigua "Europa de los quince" que no cuenta con un consejo audiovisual estatal. Nuestro país "carece de una institución destinada a velar por el cumplimiento de las normas sobre los contenidos televisivos, la publicidad, los límites a la adquisición de derechos deportivos o las obligatorias inversiones en el cine" (Gómez, 2010). Sin embargo, ya existen en España tres consejos audiovisuales de ámbito autonómico, como es el caso del Consell de l'Audiovisual de Catalunya (CAC), el Consejo Audiovisual de Navarra (COAN), y el Consejo Audiovisual de Andalucía (CAA), este último, de muy reciente creación (2013). Ahora bien, el gobierno central tiene prevista la creación del Consejo Estatal de Medios Audiovisuales (CEMA), tal y como especifica la Ley General de la Comunicación Audiovisual (LGCA) desde sus modificaciones de 2010 (El País, 01/05/2010). Tras cuatro años de extenso debate gubernamental acerca de su forma y competencias, su constitución ha quedado temporalmente apartada. El nuevo proyecto se ha inspirado en el Consejo General de lo Audiovisual Francés y estará adscrito a la Comisión Nacional del Mercado de las Telecomunicaciones (CNMT). El Consejo tendrá capacidad intervencionista y sancionadora sobre las televisiones (siendo este el aspecto más polémico). De esta forma, se pretende contar con un instrumento que contribuya a la protección de ciertos valores ciudadanos afectados por el rumbo de determinadas producciones televisivas. Sería un importante paso que contribuirá a acercarnos a Europa en cuestiones de control televisivo, cubriendo necesidades y lagunas del sistema que no han podido afrontar otras entidades ya constituidas.

\section{Materiales y metodología}

El marco teórico expuesto nos ayuda a asimilar la dificultad que para los productores de televisión actuales supone lograr, al mismo tiempo, altos niveles de audiencia y de calidad. Por esta razón, y para captar mejor la problemática a la que dichos productores se enfrentan, la presente investigación plantea la creación de un índice que permita ponderar la audiencia de las televisiones por la calidad percibida por los espectadores. Para tal fin se parte, en primer lugar, de la consideración de la cuota de pantalla por canales televisivos obtenida a partir de las bases de datos de la empresa de audiencias televisivas Kantar Media -una fuente comúnmente admitida hoy día por parte de medios e instituciones- y, en segundo lugar, del ya mencionado índice de imagen GECA por cadenas de televisión. La recogida de los datos se realiza a partir de la cuota de pantalla de los canales televisivos de junio de 2013 y de los resultados disponibles del Índice de Imagen GECA más próximo a dicha fecha (diciembre de 2012).

La cuota de pantalla queda definida en el informe técnico de Kantar Media como "el porcentaje de individuos que son audiencia de una cadena (también aplicable a 
programas o franjas) respecto al porcentaje de individuos que estaba viendo la televisión en el tiempo analizado" (Barlovento Comunicación, 2013). Por su parte, el Índice de Imagen Geca se obtiene como el promedio de las valoraciones que los espectadores entrevistados hacen de las cadenas en una escala de 1 a 10 (de la peor a la mejor nota). La cuota de pantalla se obtiene en base al panel de audiencias de Kantar Media, que coloca sus audímetros en 4.625 hogares españoles, recogiendo muestras de 11.912 sujetos mayores de cuatro años. Para su distribución, recurre a un muestreo estratificado proporcional por Comunidades Autónomas (Barlovento Comunicación, 2013). Por otra parte, el Índice de Imagen Geca se obtiene a través de 3200 entrevistas TAPI ("Tablet Assisted Personal Interview") realizadas por toda España a mayores de 14 años y en base a un muestreo aleatorio, estratificado, polietápico y por cuotas. El error muestral asumido es de +/- 1,77\% (GECA, 2012).

Como limitación importante de la propuesta, debemos resaltar que el índice de imagen GECA es una medida de calidad televisiva que se ciñe a una faceta concreta: la valoración del público de las emisiones a través de una escala de Likert, quedando por lo tanto al margen otras valoraciones de interés (técnicas y críticas) que este estudio no considera. Ahora bien, GECA considera este índice como uno de los referentes de la calidad del trabajo de las televisiones, y se ha convertido en un indicador de uso extendido en el ámbito de los estudios de comunicación para valorar la labor desempeñada por las cadenas televisivas (GECA, 2012). Asimismo, la bibliografía especializada muestra la capacidad del público para distinguir los contenidos de televisión que considera nocivos. Concretamente, el estudio de Del Moral (2012) sobre alfabetización mediática de amas de casa en edades avanzadas demuestra que estas personas distinguen los contenidos persuasivos publicitarios, así como la información manipulada y los contenidos denunciables de los programas de la televisión de masas. Sin embargo, estas muestran una actitud pasiva a la hora de movilizarse, denunciar o cambiar su conducta de consumo televisivo, lo que puede deberse en gran parte a las escasas posibilidades de elección ofrecidas para su perfil.

En base a estos planteamientos, proponemos la creación del Índice de Audiencia Relativa (IAR) para canales de televisión. Dicho índice se define como el producto de la cuota de pantalla por el índice de imagen (GECA), al que se le han restado previamente cuatro unidades. La decisión de restar cuatro puntos al índice GECA se adoptó tras aplicar pruebas de validez al nuevo índice realizando cálculos sobre la distribución completa de los canales televisivos en España. Este cambio se debe a dos razones principales: en primer lugar, permite una mejor graduación de la escala, así como una ponderación más equilibrada entre ambas variables y, en segundo lugar, no se obtuvo ni un solo resultado por debajo de 4 (escala de 1 a 10) en las valoraciones subjetivas del conjunto de los canales analizados. Una vez realizados estos ajustes, se obtiene la siguiente fórmula para el cálculo del IAR:

$$
\mathrm{IAR}=\text { Cuota de Pantalla } \mathrm{x}(\mathrm{II} \text { GECA }-4)
$$

\section{Validación del índice}

Para validar un índice de estas características, debemos recurrir a dos tipos de pruebas: pruebas de validez interna y pruebas de validez externa. 
En relación con las pruebas de validez interna, se ha calculado el índice de correlación entre las variables a partir de las cuales se obtiene el índice IAR -es decir el índice GECA y la cuota de pantalla-, considerando dos puntos temporales diferentes (test-retest: febrero-septiembre de 2012). Aunque es preferible realizar los cálculos contando con más recogidas de datos, tal opción se ha descartado por la rapidez con la que fluctúan las cadenas temáticas (muchas de ellas aparecen y desaparecen en un intervalo muy corto de tiempo), dificultando la construcción de una serie sin valores perdidos. Una vez realizados los cálculos correspondientes al coeficiente de correlación de Pearson entre la cuota de pantalla y el índice GECA, se han obtenido los siguientes valores (febrero 2012: 0,113; sig. 0,590; septiembre 2012: 0,093; sig. 0,638). Los resultados muestran en ambos puntos temporales una leve asociación positiva, aunque sin significación estadística. El resultado no dista de los planteamientos teóricos que justificaban la creación del índice: aunque la percepción de la calidad televisiva se asocia de manera positiva con la posibilidad por parte de un canal de elevar su cuota de pantalla, dicha asociación no se produce de manera estadísticamente significativa. La cuota de pantalla y la calidad percibida describen, por tanto, dos dimensiones distintas del fenómeno mediático analizado.

Por otra parte, la validez interna del índice IAR se ve reforzada por la de los indicadores que lo componen (índice GECA y cuota de pantalla), de uso generalizado y admitido en los estudios sobre medios.

En relación con las pruebas de validez externa, debemos recurrir a cuatro criterios: validación de contenido, por grupos conocidos, de construcción y predictiva:

- La validación de contenido mide la proximidad semántica entre el índice y la propiedad que se quiere medir con este. El índice IAR, como ya se ha anticipado en el planteamiento de la investigación (epígrafe 1), mide la cuota de pantalla de los canales en función de la percepción de calidad por parte de los usuarios. La toma en consideración de dichas dos variables para el cálculo del índice nos acerca a la propiedad "audiencia relativa", o nivel de audiencia generado en función de la calidad percibida por el espectador. Recuérdese que su utilidad se basa en la dificultad de generar alta audiencia cuidando al mismo tiempo la calidad en el sistema actual.

- El segundo criterio a satisfacer, la validación por grupos conocidos, permite controlar que los valores obtenidos por el índice guarden cierto grado de correspondencia con otras mediciones de indicadores conceptualmente próximos. Dada la carencia de dimensiones similares en investigación de audiencias, nuestra tarea se limita a comprobar los comportamientos dispares que, con cierta frecuencia, se dan en el mercado televisivo entre audiencia y calidad, tal y como se podrá apreciar en el apartado 5, correspondiente al análisis de los resultados.

- La validación por construcción exige responder, a su vez, a cuatro criterios distintos: ausencia de valores perdidos, estandarización y normalización, misma direccionalidad y ponderación. 
Tabla 1: Cuota de pantalla e índice GECA de las principales cadenas televisivas en 2012. Fuente: Elaboración propia a través de datos de GECA y Kantar Media.

\begin{tabular}{|l|l|r|r|r|}
\hline Canales TV & Clasificación & \multicolumn{2}{|c|}{$\begin{array}{c}\text { Cuota pantalla } \\
\text { feb. 2012 }\end{array}$} & \multicolumn{2}{c|}{$\begin{array}{l}\text { Cuota pantalla } \\
\text { sept. 2012 }\end{array}$} & $\begin{array}{l}\text { Índice } \\
\text { GECA 2012 }\end{array}$ \\
\hline Tve 1 & Generalista & 14,10 & 11,20 & 6,70 \\
\hline Antena 3 & Generalista & 12,20 & 12,70 & 6,80 \\
\hline Telecinco & Generalista & 13,70 & 13,80 & 5,90 \\
\hline Cuatro & Generalista & 6,30 & 5,90 & 6,70 \\
\hline Sexta & Generalista & 4,80 & 5,00 & 6,60 \\
\hline Forta & Generalista & 10,40 & 9,40 & 6,30 \\
\hline La 2 & Generalista & 2,70 & 2,30 & 6,30 \\
\hline Neox & Temático & 2,70 & 2,60 & 6,80 \\
\hline Nova & Temático & 1,30 & 1,60 & 6,30 \\
\hline Nitro & Temático & 1,50 & 1,50 & 6,40 \\
\hline FDF & Temático & 2,60 & 3,30 & 6,70 \\
\hline Clan & Temático & 2,40 & 2,80 & 6,50 \\
\hline Disney & Temático & 1,60 & 1,70 & 6,30 \\
\hline La Sexta 3 & Temático & 1,50 & 1,80 & 6,80 \\
\hline TDP & Temático &, 60 &, 70 & 6,70 \\
\hline 24 horas & Temático &, 90 &, 90 & 6,70 \\
\hline Boing & Temático & 1,40 & 2,00 & 6,10 \\
\hline Divinity & Temático & 1,30 & 1,50 & 6,30 \\
\hline Siete & Temático & 1,40 & 1,40 & 6,30 \\
\hline Discovery & Temático & 1,20 & 1,30 & 6,90 \\
\hline Marca & Temático &, 80 & 1,00 & 6,20 \\
\hline Intereconomía & Temático & 1,50 & 1,10 & 4,50 \\
\hline 13 & Temático &, 80 & 1,00 & 5,60 \\
\hline Energy & Temático & Temático & & 6,30 \\
\hline MTV & & & 6,50 \\
\hline
\end{tabular}

1. Ausencia de valores perdidos: las series analizadas tienen que carecer, en la medida de lo posible, de valores perdidos. Para el cumplimiento del criterio se escogieron dos puntos temporales que permitieran escasas variaciones en la parrilla en cuanto a oferta de canales (febrero y septiembre de 2012), tal y como se aprecia en la Tabla 1 .

2. Normalización: se han aplicado pruebas de normalización estadística al IAR, tanto en la serie de canales generales y temáticos, como en la conjunta. La clasificación y la separación de ambos tipos de canales para realizar comparaciones es obligada, dadas las amplias diferencias que, hoy en día, existen entre las cuotas de pantalla de unos y otros (público de masas frente a público segmentado). Por ejemplo, mientras un 4\% puede implicar un enorme fracaso para una gran cadena generalista, podría significar un gran éxito para una temática. La serie de canales temáticos analizada muestra una distribución cercana a la normal, como se puede apreciar en el Gráfico 1 (media: 3,57; desviación estándar: 1,77, $\mathrm{n}=21$ ). Los canales generalistas muestran una tendencia similar, aunque no se considera la significación por tratarse de una serie muy reducida. Asimismo, las pruebas realizadas sugieren que no procede realizar mediciones conjuntas por la gran disparidad en los valores que se obtienen entre cadenas generalistas y temáticas: las cuotas de pantalla de ambas son muy diferentes y, por lo tanto, también ocurre con su IAR. 


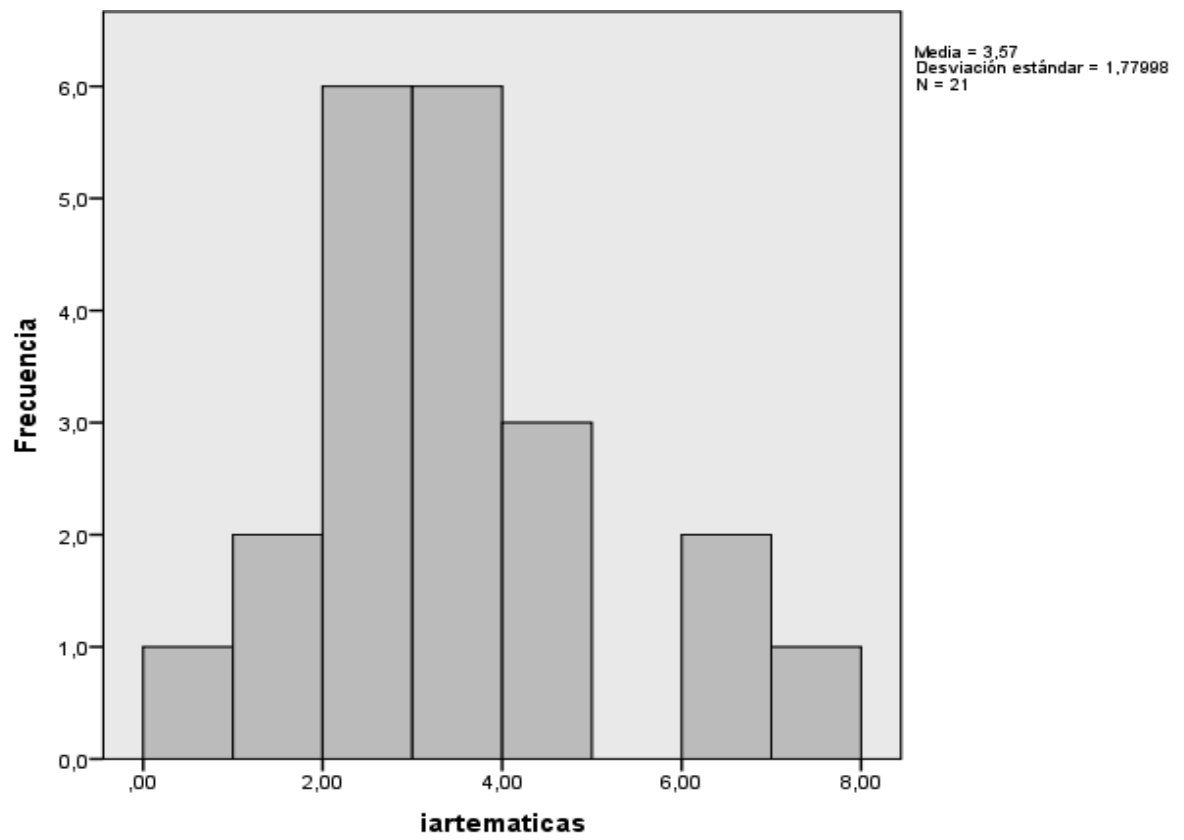

Gráfico 1. Distribución de la variable IAR correspondiente a las cadenas televisivas temáticas

3. Misma direccionalidad: las dos variables que componen el índice se manifiestan en una misma dirección (índice GECA y cuota de pantalla).

4. Ponderación: se ha tratado de evitar la sobrerrepresentación de una variable sobre la otra. Para ello, como ajuste previo para el índice GECA, cuyos valores se concentraban entre 4,5 y 6,9 (escala de 1 a 10), se optó por prescindir de los 4 primeros grados (GECA - 4). Este no es el caso de la cuota de pantalla, que presenta grandes diferencias entre mínimo y máximo $(0,1-14,3)$, y un alto grado de dispersión en sus datos.

En resumen, las pruebas muestran que el comportamiento estadístico del IAR apoya su validez interna, mientras que en validez externa presenta algunas dificultades, fundamentalmente por la carencia de registros de dimensiones precedentes que midan la misma propiedad o se aproximen. Esto, a su vez, es consecuencia de su propia concepción: generar un índice que permita la medida de una dimensión no recogida en estudios precedentes, aportando una novedad al campo.

Llevado el índice a un plano práctico, planteamos la hipotética necesidad de comparar un canal temático del grupo Mediaset (Boing) con otro de Atresmedia (Xplora), en su desempeño tanto en cuota de pantalla como en audiencia relativa; para esto, debemos considerar sus cuotas de pantalla (obtenidas a partir de Kantar Media) en 2012 (1,8\% y $1,7 \%$, respectivamente), además de sus índices de imagen GECA (6,1 y 6,9, respectivamente). Después, al calcular los respectivos IAR, se obtiene lo siguiente:

$$
\begin{array}{ll}
\text { IAR Boing }= & 1,8 \times(6,1-4)=3,78 \\
\text { IAR Xplora }= & 1,7 \times(6,9-4)=4,93
\end{array}
$$


En este caso, podemos observar el comportamiento de dos canales con cuota de pantalla muy similar $(1,8 / 1,7)$; sin embargo, al ponderarse esta con los resultados del índice GECA -más alto en el caso de la cadena Xplora-, dicho canal obtiene un valor final mayor que Boing en el IAR (4,93 frente a 3,78).

En definitiva, gracias al cálculo del IAR logramos ponderar la audiencia en función de un indicador de calidad, de forma que, en una potencial clasificación, las cadenas con mayor cuota de pantalla no mantendrán su posición en este indicador si no mantienen, al mismo tiempo, razonables niveles en imagen. A continuación, el índice será aplicado a los grupos televisivos Mediaset y Atresmedia, comparando tanto sus canales de referencia como los temáticos y sus cifras agregadas.

\section{Resultados y análisis}

5.1. Descripción previa del panorama televisivo en audiencia e índice de imagen Aunque nuestro objetivo fundamental consiste en comparar el grupo Atresmedia con el grupo Mediaset, resulta ineludible una primera aproximación a la distribución de la cuota de pantalla de los grandes canales generalistas -tanto públicos como privados(Cuadro 1), así como al índice de imagen GECA que obtiene cada uno de ellos (Cuadro 2). De esta forma, podemos observar el actual dominio en audiencia de las cadenas emblemáticas de los dos grupos mencionados -Telecinco y Antena 3, con un 14,5\% y un $12,9 \%$, respectivamente, en junio de 2013- sobre los canales públicos. Esta tendencia se ha visto facilitada por un importante descenso de la audiencia de TVE1 en los últimos tiempos: 9,6\% en junio de 2013, cuando había llegado, en 2011, a acercarse al $17 \%$. Además, La 2 de TVE ha experimentado un destacado descenso (1legando a solo el 2,3\% en junio de 2013). En definitiva, la televisión pública ha sufrido notoriamente la competencia de los dos grandes grupos privados, que han diversificado sus productos con la implantación del sistema digital. En cuanto al índice de imagen, Antena 3, TVE 1 y Cuatro ocupan las tres primeras posiciones, con calificaciones casi idénticas $(6,8,6,7$ y 6,7$)$, mientras que la última posición de Telecinco $(5,9)$ llama la atención, ya que sus propios televidentes muestran una baja percepción de la calidad de unas emisiones que, no obstante, continúan siguiendo. En este ámbito, Telecinco ha visto descender su valoración subjetiva desde el año 2009, cuando alcanzó una puntuación de 6,8; además, la pérdida de cuatro décimas respecto al año anterior (6,3 en 2011) parece haber incrementado el ritmo de tal decrecimiento. En definitiva, el conjunto de los resultados nos permite subrayar el bajo grado de asociación existente entre la audiencia de nuestros canales de televisión y la valoración subjetiva obtenida por estas.

Tabla 2. Audiencias recientes de las cadenas generalistas en España ${ }^{1}$

\begin{tabular}{lrr}
\hline \hline $\begin{array}{c}\text { CADENAS } \\
\text { (GENERALISTAS) }\end{array}$ & $\begin{array}{c}\text { AUDIENCIA } \\
\text { JUNIO 2013 }\end{array}$ & $\begin{array}{c}\text { AUDIENCIA } \\
\text { JUNIO 2012 }\end{array}$ \\
\hline \hline TELECINCO & $14,5 \%$ & $14,7 \%$ \\
ANTENA 3 & $12,9 \%$ & $12,0 \%$ \\
LA 1 & $9,6 \%$ & $11,9 \%$ \\
AUTONÓMICAS & $8,6 \%$ & $9,5 \%$
\end{tabular}

${ }^{1}$ Elaboración propia en base a datos recogidos por Barlovento Comunicación. 


\begin{tabular}{lrr} 
CUATRO & $6,4 \%$ & $7,5 \%$ \\
LA SEXTA & $6,2 \%$ & $4,8 \%$ \\
LA 2 & $2,3 \%$ & $2,3 \%$ \\
\hline
\end{tabular}

Tabla 3. Índice de Imagen GECA de las principales cadenas generalistas

\begin{tabular}{|c|c|}
\hline $\begin{array}{c}\text { CADENAS } \\
\text { (GENERALISTAS) }\end{array}$ & $\begin{array}{c}\text { Puntuaciones de } \\
\text { los entrevistados (1-10) }\end{array}$ \\
\hline ANTENA 3 & 6,8 \\
\hline LA 1 & 6,7 \\
\hline CUATRO & 6,7 \\
\hline LA SEXTA & 6,6 \\
\hline AUTONÓMICAS & 6,3 \\
\hline LA 2 & 6,3 \\
\hline TELECINCO & 5,9 \\
\hline
\end{tabular}

Si sometemos a comparación los canales temáticos de los dos grandes grupos en audiencia e índice de imagen GECA en las mismas fechas (Cuadro 3), observamos que la situación está equilibrada en cuota de pantalla, aunque con una leve ventaja por parte del grupo Mediaset (9,7\% frente al 8,6\% de Atresmedia), gracias particularmente al éxito de la cadena Factoría de Ficción (FDF), que aporta un 2,9\%. Sin embargo, las cadenas temáticas de Atresmedia se encuentran algo mejor situadas que su gran competidor en el índice de imagen, ya que dicho grupo ha conseguido colocar tres de sus canales entre los cuatro primeros de la clasificación global de canales temáticos (Xplora, La Sexta 3 y Neox, entre el segundo y el cuarto puesto, siendo el líder Discovery Max, de TVE). Por el contrario, Mediaset no ha podido ubicar a sus cadenas temáticas entre las primeras clasificadas en índice de imagen GECA. En este sentido, resulta llamativo que FDF, a pesar de su peso en la cuota de pantalla, no haya podido superar un discreto sexto lugar, en una nueva muestra de la disparidad existente entre los resultados en audiencia y la valoración del espectador.

Tabla 4. Posiciones de las cadenas temáticas de Atresmedia y Mediaset en cuota de pantalla e Índice de Imagen GECA ${ }^{2}$

\begin{tabular}{|c|c|c|c|c|c|}
\hline CANAL & GRUPO & $\begin{array}{l}\text { AUDIENCIA } \\
\text { JUNIO } 2013\end{array}$ & $\begin{array}{l}\text { AUDIENCIA } \\
\text { TEMÁTICAS }\end{array}$ & $\begin{array}{c}\text { ÍNDICE } \\
\text { GECA }\end{array}$ & $\begin{array}{c}\text { GECA } \\
\text { TEMÁTICAS }\end{array}$ \\
\hline FDF & Mediaset & 2,9 & $1^{\circ}$ & 6,7 & $6^{\circ}$ \\
\hline NEOX & Atresmedia & 2,4 & $3^{\circ}$ & 6,8 & $4^{\circ}$ \\
\hline NOVA & Atresmedia & 1,9 & $4^{\circ}$ & 6,3 & $14^{\circ}$ \\
\hline BOING & Mediaset & 1,8 & $5^{\circ}$ & 6,1 & $18^{\circ}$ \\
\hline XPLORA & Atresmedia & 1,7 & $6^{\circ}$ & 6,9 & $2^{\circ}$ \\
\hline NITRO & Mediaset & 1,6 & $7^{\circ}$ & 6,4 & $11^{\circ}$ \\
\hline DIVINITY & Mediaset & 1,6 & $9^{\circ}$ & 6,3 & $15^{\circ}$ \\
\hline LASEXTA3 & Atresmedia & 1,6 & $10^{\circ}$ & 6,8 & $3^{\circ}$ \\
\hline ENERGY & Mediaset & 1,2 & $14^{\circ}$ & 6,3 & $12^{\circ}$ \\
\hline NUEVE & Mediaset & 0,7 & $17^{\circ}$ & - & - \\
\hline
\end{tabular}

2 Elaboración propia en base a datos recogidos por GECA y Barlovento Comunicación. 
En cuanto a la cuota de pantalla agregada obtenida por los dos grandes grupos de comunicación del país (Cuadro 4), según datos de junio de 2013, el grupo Mediaset logró un 30,5 \% (Grupo Telecinco: 21,2 \% y Grupo Cuatro: 9,3 \%), mientras que Atresmedia ha obtenido un 28,5\% (agrupando sus empresas A3 Cobertura: 19,9\% y A3 Afinidad: 8,6\%). Ambas entidades mantienen una notable distancia con el líder del sector público, TVE, que obtuvo un $16,1 \%$ entre todos sus productos. Por lo tanto, nos encontramos ante una situación de equilibrio entre las dos grandes firmas privadas, que se refleja también en la cuota de pantalla de sus respectivos canales generalistas (14,5\% de Telecinco, frente a casi un 13\% de Antena 3). De cualquier forma, queda patente el dominio de la audiencia actual de los dos grandes grupos privados, dejando atrás el sector público.

\begin{tabular}{|c|c|}
\hline $\begin{array}{l}\text { PRINCIPALES GRUPOS } \\
\text { TELEVISIVOS }\end{array}$ & $\begin{array}{c}\text { CUOTA DE PANTALLA } \\
\text { (junio 2013) }\end{array}$ \\
\hline ANTENA 3 & 19,9 \\
\hline TELECINCO & 21,2 \\
\hline A3 AFINIDAD & 8,6 \\
\hline CUATRO & 9,3 \\
\hline TVE & 16,1 \\
\hline MEDIASET (TOTAL) & 30,5 \\
\hline ATRESMEDIA (TOTAL) & 28,4 \\
\hline
\end{tabular}

\subsection{Aplicaciones del Î́ndice de Audiencia Relativa}

A continuación, procedemos a calcular el nuevo indicador (IAR) aplicando la fórmula ya descrita en la metodología (apartado 3) a los canales de televisión generalistas:

IAR $=$ Cuota de Pantalla $\times($ II GECA -4$)$

Como se puede apreciar en el Gráfico 2, encontramos a los productos más emblemáticos de los dos grandes grupos privados en los primeros puestos. Entre ellos, Antena 3 obtiene un IAR superior al de Telecinco (36,12 frente a 27,55), a pesar de haber logrado una cuota de pantalla algo inferior a la de la cadena de Mediaset. En tercer lugar, y muy cerca de Telecinco, aparece la gran cadena de la televisión pública, TVE1 $(26,1) \mathrm{y}$, posteriormente, encontramos escasas distancias entre el bloque de las Autonómicas $(19,78)$, Cuatro (segundo canal en audiencia de Mediaset, con 17,28) y La Sexta (perteneciente a Atresmedia, con 16,12). El dominio de Antena 3 en esta clasificación es la consecuencia lógica de su segundo puesto en cuota de pantalla y de su primer puesto en valoración GECA.

3 Elaboración propia en base a datos recogidos por Barlovento Comunicación. 


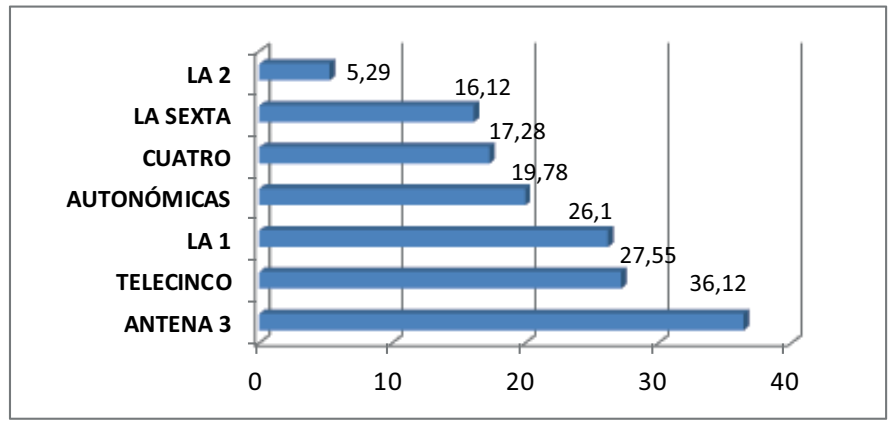

Gráfico 2. Indice de Audiencia Relativa (junio 2013) de las principales cadenas de televisión generalista ${ }^{4}$

En una segunda aplicación del IAR, sometemos a comparación los canales temáticos de los dos grandes grupos (Gráfico 2). Fdf(Mediaset) ocupa el primer puesto con un IAR de 7,83. Le siguen los cuatro productos más destacados del grupo Atresmedia: Neox $(6,72)$, Xplora $(4,93)$, La Sexta $3(4,48)$ y Nova $(4,37)$, que superan a las restantes temáticas de Mediaset: Nitro $(3,84)$, Boing $(3,78)$ y Divinity $(3,68)$. Debemos resaltar que los valores obtenidos por Fdf y Neox son particularmente altos para tratarse de canales temáticos. Concretamente, ambos superan en IAR a un canal generalista de amplia tradición en la televisión pública como La 2 de TVE $(5,29)$. Esto se debe a que su valoración en índice de imagen es mayor que el de los canales generalistas mejor puntuados (6,7 en Fdf y 6,8 en Neox). Esta clasificación varía levemente respecto a la de las audiencias. En ella, Fdf y Neox ocupan los dos primeros puestos; sin embargo, La Sexta 3 ocupa el último lugar y Boing, el cuarto puesto; es decir, al obtener el IAR, mejora la posición de La Sexta 3, mientras que empeora en el caso de Boing.

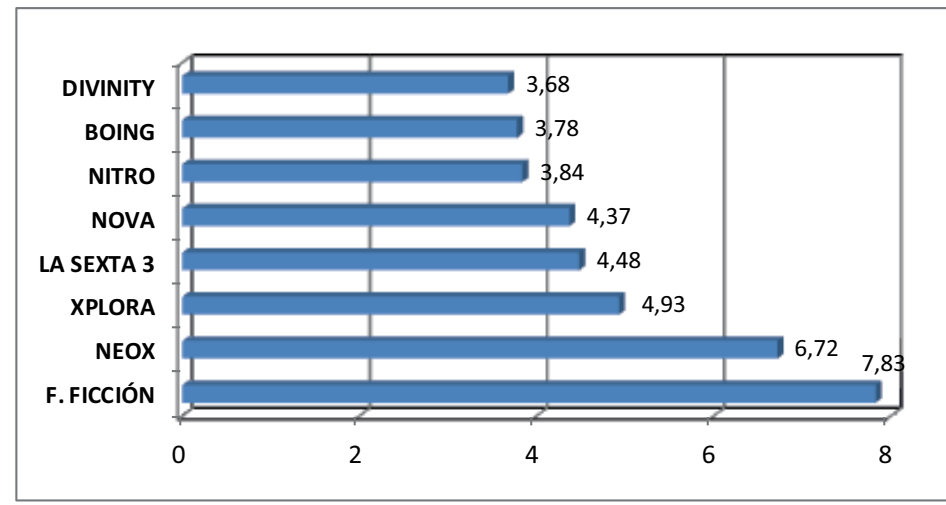

Gráfico 3. Índice de Audiencia Relativa (junio, 2013) de las principales cadenas temáticas de televisión: Mediaset y Atresmedia

${ }^{4}$ Elaboración propia en base a datos recogidos por GECA y Barlovento Comunicación. 
$\mathrm{Al}$ calcular el IAR que promedian ambos grupos al agregar sus canales generalistas y temáticos, el grupo Atresmedia se presenta levemente por encima de Mediaset $(11,3$ frente a 10,6). En definitiva, unas valoraciones más altas en el índice de imagen GECA en los canales de Atresmedia le permiten superar en IAR a Mediaset. Por el contrario, la situación de las audiencias globales de ambos grupos se decanta en favor de Mediaset, con una corta ventaja de dos puntos sobre Atresmedia (30,5\% frente a 28,4\%, según datos de Kantar Media en junio de 2013).

En definitiva, el panorama televisivo español presenta una cuota de pantalla dominada por las dos grandes corporaciones privadas, en detrimento de la posición de las entidades públicas. El retroceso de este sector como consecuencia del empuje de los grandes grupos privados tiene una muestra palpable en la situación de La 2: después de superar el $7 \%$ en cuota en los primeros 2000 , a verse superada por canales temáticos.

En la actualidad, entre las dos grandes corporaciones privadas se da una situación de equilibrio, con una leve diferencia (2\%) a favor de Mediaset sobre Atresmedia (junio, 2013). Sin embargo, ambas entidades están generando su audiencia con diferencias importantes en los niveles de valoración de sus espectadores, contando Atresmedia con una posición notablemente mejor, tal y como queda manifiesto en el Índice de Imagen GECA 2012.

\section{Conclusiones}

La construcción del Índice de Audiencia Relativa ha facilitado la comparación de la producción televisiva de las dos grandes corporaciones privadas del país (Atresmedia y Mediaset) considerando, al mismo tiempo, sus cuotas de pantalla y sus índices de imagen GECA respectivos. De esta forma, el IAR ha llevado a la ponderación de dos variables fundamentales que, dada la aproximación teórica realizada (Apartados 1 y 2), pretendían medirse de manera conjunta. Atresmedia obtiene un IAR más alto que Mediaset, considerando los datos actuales. Este resultado se produce después de que, desde el año 2009, el grupo difundiera sus pretensiones de dar un giro hacia la calidad, tratando de escapar del etiquetaje de "amarillismo televisivo" y "fomentando una cultura televisiva que apuesta por programas para toda la familia" (Vallejo Peña: 2012: 113). Esto último, sin renunciar a políticas orientadas a aumentar su audiencia y cuota de pantalla (ha subido paulatinamente desde entonces hasta 2013). Mediaset, por su parte, se ha situado por debajo de seis puntos en el índice de imagen GECA en 2012 $(5,9) \mathrm{y}$, como consecuencia, presenta un IAR inferior al de Atresmedia. Este aspecto, sin embargo, no cuestiona el liderazgo de Mediaset en el sector, avalado por su cuota de pantalla.

Los resultados obtenidos en este trabajo nos conducen, además, a reflexionar sobre la relevancia social que tendría el hecho de que las dos grandes corporaciones citadas tuvieran cada vez más en cuenta la calidad de sus producciones. Entre ambas agrupan un $58,9 \%$ de la cuota de pantalla nacional, con la influencia que esto supone sobre la socialización, la educación y el sistema de valores del país. Se trata de una recreación más de un fenómeno ya vaticinado por las tesis elaboradas en los años setenta por Lazarsfeld y Merton (Lazarsfeld et al., 1977). La progresiva transformación del modelo 
televisivo y su reconversión a un producto de corte más social, que ayudara a recuperar su carácter de servicio público, pasaría por una reformulación de los objetivos de estas dos grandes empresas.

En el caso español, tanto la revisión teórica como los datos presentados en el análisis sugieren que tendemos a ver la misma televisión que frecuentemente censuramos (Del Moral, 2012). De hecho no existe una relación estadísticamente significativa entre la variación en la calidad televisiva percibida y los cambios en la cuota de pantalla. El estudio señalado sobre las competencias audiovisuales de los españoles (Lazo, 2013), corrobora un escenario de indefensión de un público dócil y manipulable ante los medios, al no haber desarrollado capacidades propias de una audiencia activa, participativa y facultada para decodificar los mensajes recibidos y las emociones representadas.

Por lo tanto, la presión de los agentes y las exigencias en responsabilidad social deben llevar a los grandes grupos a conjugar la audiencia que logran con "el cómo" la logran. Lo realmente difícil para ellos no es acceder a las audiencias de masas, sino lograrlo bajo unos mínimos criterios de calidad. El giro reciente de Atresmedia hacia una televisión socialmente más constructiva, impulsada por un cambio en su programa de Responsabilidad Social Corporativa, es un paso interesante y todo un precedente para la televisión privada y su futuro desempeño. Estas circunstancias sugieren que el cuidado de la calidad de la televisión y su contribución social puede y debe convertirse en una preocupación privada. El modelo actual genera externalidades negativas que acaban dañando los intereses de la mismos productores: "La cadena (Telecinco) -ubicada en los últimos lugares por el índice de imagen GECA- ha sacrificado incluso su imagen pública por los mencionados logros (en audiencia)" (Vallejo Peña, 2012: 119). De hecho, más allá de las meras cuestiones de imagen, en 2011 la cadena tuvo que afrontar sentencias judiciales por gastos superiores a dos millones de euros.

Una vez subrayada la necesidad de contrarrestar la tendencia televisiva a dominar las audiencias con el criterio del máximo común denominador, se debe incidir en los instrumentos. La presente propuesta metodológica pretende, con la aportación del IAR, la proposición de una alternativa social y empresarialmente viable. De cualquier forma, investigadores, administraciones públicas, asociaciones ciudadanas, consumidores e incluso los propios medios necesitan referencias que les permitan calificar sus productos en base a la respuesta social (valoración) de sus emisiones, más allá de la mera medición de las audiencias captadas.

Ante la dureza del escenario planteado, la presumible creación del Consejo Estatal de los Medios Audiovisuales (CEMA) por parte del gobierno de la nación -dependiente de la Comisión del Mercado de las Telecomunicaciones (CMT)- llegará un tanto tarde para el único gobierno de la antigua "Europa de los quince" que no cuenta con tal organismo. No obstante, se presenta como una oportunidad para que el Estado ejerza mayor control e intervención sobre los contenidos de las televisiones privadas. Dicho Consejo -con potestad sancionadora- puede contribuir a proteger nuestros derechos, así como cuidar la difusión mediática de los valores entre los ciudadanos. En la calidad de la televisión que ven nuestros niños y adolescentes, y en la conservación de su rol de servicio público está, en gran medida, el futuro de España. 


\section{Agradecimientos}

Agradecimientos: a Sandro Giachi (IESA-CSIC) y a Verónica de Miguel Luken (UMA) por su asesoramiento estadístico.

\section{Referencias bibliográficas}

BARLOVENTO COMUNICACIÓN (2013): Comportamiento de la audiencia televisiva. Madrid, Barlovento.

BAUDRILLARD, Jean (1978): Cultura y simulacro. Barcelona, Kairós.

BERGER, Peter \& LUCKMANN, Thomas (1967): The social construction of reality. London, Penguin books.

BORDAS, Julio (2005): "La sociedad caleidoscópica actual”. Documentación Social, no 139, pp. 193-209.

BOURDIEU, Pierre (1998): On Tv. New York, The New Press.

BUENO, Gustavo (2003): Telebasura y democracia. Madrid, Punto de Lectura.

BUSTAMANTE, Enrique (1999): La televisión económica: financiación, estrategias y mercados. Barcelona, Gedisa.

COLEMAN, James (1990): Foundations of Social Theory. Cambridge, Harvard University Press.

DEL MORAL, María Esther y VILLALUSTRE, Lourdes (2012): “Alfabetización mediática, participación y responsabilidad frente a los medios de la generación del silencio". Comunicar, no 40, pp. 173-181.

DE MORAGAS, Miguel (1982): Sociología de la comunicación de masas. Barcelona, Gustavo Gili.

DOYLE, Gillian (2002): Understanding media economics. London, Sage.

ELÍAS, Carlos (2004): Telebasura y periodismo: se deslizan las fronteras. Madrid, Libertarias.

FOG, Agner (2004): "The supposed and the real role of mass media in modern democracy", en Agner Fog: http://www.agner.org/cultsel/mediacrisis.pdf [Consulta: 20 de enero de 2013].

GARCÍA FERRANDO, Manuel (1998): Sociología del deporte. Madrid, Alianza.

GECA (Ed., 2012): Estudio de imagen GECA 2012. Madrid, Grupo de Estudios de Comunicación Audiovisual.

GÓMEZ, Rafael (2010): "Luz verde para la creación de un órgano regulador en televisión", en El País, 18 de marzo, sección Sociedad.

GRANDÍO, María del Mar (2009): "El entretenimiento televisivo. Un estudio de audiencia desde la noción del gusto". Comunicación y sociedad, vol. XXII, $\mathrm{n}^{\mathrm{o}} 2$, pp.139-158.

IBÁÑEZ, Jesús (1985): Del algoritmo al sujeto. Madrid, Siglo XXI. 
KATZ, Elihu, GUREVITCH, Michael y HAAS, Hadassah (1973): "On the use of the mass media for important things". American Sociological Review, n ${ }^{\circ} 38$, pp. $164-$ 181.

LACALLE (2000), María Rosario (2000): "Mitologías cotidianas y pequeños rituales televisivos. Los talk shows". Anàlisi, n 24, pp. 79-92.

LAZARSFELD, Paul y MERTON, Robert (1977): "Comunicación de masas, gusto popular y acción social organizada", en MURARO, Heriberto: La comunicación de masas. Buenos Aires, Ceal, pp. 25-47.

MARTA LAZO, Carmen y GRANDÍO, María del Mar (2013): “Análisis de la competencia audiovisual de la ciudadanía española en la dimensión de recepción y audiencia”. Comunicación y sociedad, vol. XXVI, nº 2, pp. 114-130.

McMANUS, John (1995): “A market-based model of news production”. Communication Theory, $\mathrm{n}^{\circ}$ 5, pp. 331-338.

MURRAY, Susan \& OUELLETTE, Laura (2009): Reality TV: Remaking Television Culture. New York, New York University Press.

ORTIZ, Miguel Ángel; RUIZ, Juan Antonio; y DÍAZ, Elba (2013): “¿Están las cadenas de televisión interesadas en una mejor TV para los menores? Las televisiones y la investigación en infancia y televisión". Comunicar, n 40, pp. 137-144.

RAMONET, Ignacio (1998): La tiranía de la comunicación. Madrid, Debate.

VALLEJO PEÑA, Alberto (2012): "Medios de comunicación de masas y sistema de valores: un acercamiento al programa de Responsabilidad Social Corporativa de Telecinco". Revista de Responsabilidad Social de la Empresa, nº 12, pp. 91-124.

VARIAN, Hal (2005): Microeconomía intermedia: un enfoque actual. Barcelona, Antoni Bosch.

VILLENA, Andrés (2005): "La explotación del invitado en los programas de testimonios o talk shows: el caso de El diario de Patricia". Ponencia en Foro Universitario de Investigación en Comunicación, Madrid. 